\title{
A note on the bottom shear stress in oscillatory planetary boundary layer flow
}

\section{DAG MYRHAUG $\dagger$}

\begin{abstract}
Keywords: Oscillatory planetary flow, boundary layer, eddy viscosity, bottom shear stress.

A simple analytical theory is presented, which describes the motion in a turbulent oscillatory planetary boundary layer near a rough seabed using a two-layer, time-invariant eddy viscosity model. The bottom shear stress is outlined, and comparison is made with Pingree and Griffiths' (1974) measurements of turbulent tidal planetary boundary layer flow on the continental shelf south-west of Lands End, England.
\end{abstract}

\section{Introduction}

The vertical structure of the bottom boundary layer on continental shelves is dominated by several interacting physical effects in the most widespread, complex case. Among these effects are the earth's rotation, tidal effects, stratification due to salinity and temperature gradients and suspended sediments, internal friction in the fluid and topographical effects.

One important feature of the vertical structure of the bottom boundary layer is determined by the influence of planetary rotation on various types of flow such as unsteady flow due to tidal effects, horizontally uniform flow, and unbounded and unstratified flow. This idealized boundary layer flow may occur in the ocean away from any coasts and in the region near the seabed where the internal friction in the fluid is significant.

The details of the vertical structure of the current varies with the modelling of the momentum flux in the boundary layer. Various types of models have been presented in Sverdrup (1927), Long (1981), Prandle (1982), Davies (1985) and King et al. (1985) among others. Soulsby (1983) gives a review of bottom boundary layers of shelf seas. Some of the idealized types of boundary layer situations were discussed using an eddy viscosity model which varies linearly with the distance from the bottom.

This paper presents an analytical theory which describes the fluid motion in a turbulent oscillatory planetary boundary layer near a rough seabed. A two-layer, time-invariant eddy viscosity model is used to model the shear stress. The eddy viscosity in the inner layer increases quadratically with the distance from the bottom. In the outer layer, the eddy viscosity is taken as a constant. The present eddy viscosity model is a reasonable compromise between accuracy and simplicity and has an advantage over the linear profile that there is one disposable constant which enables the model to be adjusted to the data. The choice of this constant determines the magnitude of the eddy viscosity in the outer layer, as well as the

Received 5 November 1987.

$\dagger$ Division of Marine Hydrodynamics, Norwegian Institute of Technology, N-7034 Trondheim-NTH, Norway. 
height of the overlap point. The bottom shear stress is presented and a comparison is made with measurements of turbulent tidal planetary boundary layer flow on the continental shelf south-west of Lands End, England reported by Pingree and Griffiths (1974). The estimated bottom shear stress from these measurements agrees very well with the predictions made by our simple eddy viscosity model.

It should be noted that the present theory is valid for current without any influence of wave action. Application of this theory to time-dependent currents induced by wind stress will therefore be restricted to situations when no waves are present. However, Jenkins (1987) has most recently presented a theory for time-dependent currents induced by a variable wind stress and wave field in deep water away from coastal boundaries.

\section{The oscillatory planetary boundary layer model}

When studying first-order marine boundary layer problems it is common to neglect vertical components of velocity, convective acceleration terms and density effects. The time-dependent equations of motion along the horizontal orthogonal axes $x$ and $y$ (east and north, respectively, in the northern hemisphere) can be combined and expressed in complex notation by (Soulsby 1983).

$$
\frac{\partial R}{\partial t}+\mathrm{i} f R=-g S+\frac{1}{\rho} \frac{\partial T}{\partial z}
$$

where $\mathrm{i}=(-1)^{1 / 2}$ and the following complex notation has been introduced for the velocity, the water surface slope and the frictional shear stress, respectively,

$$
\begin{aligned}
& R=U+i V \\
& S=\frac{\partial \zeta}{\partial x}+\mathrm{i} \frac{\partial \zeta}{\partial y} \\
& T=\tau_{x z}+i \tau_{y z}
\end{aligned}
$$

where $z$ is the vertical axis measured upwards in a positive direction from the seabed, $U$ and $V$ are velocities along $x$ and $y, g$ is the acceleration of gravity, $\zeta$ is the water surface elevation, $\rho$ is the density of the fluid, $\tau_{x z}$ and $\tau_{y z}$ are the $x$ - and $y$-components of the frictional shear stress. $f=2 \Omega \sin \psi$ is the Coriolis parameter, where $\Omega$ is the Earth's angular frequency of rotation and $\psi$ the latitude. The flow is driven by the horizontal pressure gradient due to the horizontal time-varying water surface slope.

On the seabed the velocity is zero and outside the boundary layer the frictional shear stress is negligible. Thus the boundary conditions are given by

$$
\begin{gathered}
R=0 \text { at } \quad z=z_{0} \\
R \rightarrow R_{\infty} \text { for } \quad z \rightarrow \infty
\end{gathered}
$$

where $z_{0}$ is the seabed roughness length and $R_{\infty}$ is the free stream velocity outside the boundary layer.

For harmonic time-dependent oscillations with angular frequency $\omega$, the complex velocity in Eqn. (2) can be written as (Soulsby 1983)

$$
R=R_{+} \exp (\mathrm{i} \omega \mathrm{t})+R_{-} \exp (-\mathrm{i} \omega t)
$$


where

$$
\begin{aligned}
& R_{+}=\left|R_{+}\right| \exp \left(\mathrm{i} \phi_{R_{+}}\right)=\frac{1}{2}\left[\left(a_{U}+b_{V}\right)+\mathrm{i}\left(a_{V}-b_{U}\right)\right] \\
& R_{-}=\left|R_{-}\right| \exp \left(\mathrm{i} \phi_{R_{-}}\right)=\frac{1}{2}\left[\left(a_{U}-b_{V}\right)+\mathrm{i}\left(a_{V}+b_{U}\right)\right]
\end{aligned}
$$

and

$$
\begin{array}{ll}
a_{U}=U_{0} \cos \phi_{U}, & b_{U}=U_{0} \sin \phi_{U} \\
a_{V}=V_{0} \cos \phi_{V}, & b_{V}=V_{0} \sin \phi_{V}
\end{array}
$$

Here $U_{0}, V_{0}$ and $\phi_{U}, \phi_{V}$ are the amplitudes and phases of $U$ and $V$, respectively. The velocity has been divided into anticlockwise (Eqn. (7)) and clockwise (Eqn. (8)) rotations. $R_{+}$represents a velocity vector with magnitude $\left|R_{+}\right|$and phase $\phi_{R_{+}}$ rotating anticlockwise with frequency $\omega$, when viewed from above. $R_{-}$represents a velocity vector with magnitude $\left|R_{-}\right|$and phase $\phi_{R_{-}}$, this rotates clockwise with frequency $\omega$. Thus the combination of the two rotations in Eqn. (6) represents a velocity vector which describes an ellipse, see Fig. 1. Here the maximum and minimum currents each correspond to the semi-major and semi-minor axes of the ellipse, and are given by

$$
\begin{aligned}
& |R|_{\text {max }}=\left|R_{+}\right|+\left|R_{-}\right| \\
& |R|_{\text {min }}=\left\|R_{+}|-| R_{-}\right\|
\end{aligned}
$$

respectively. The time phase of maximum current is given by

$$
\theta=(\omega t)_{\max }=\frac{\phi_{R_{-}}-\phi_{R_{+}}}{2}
$$

while the direction of the maximum current (the orientation of the semi-major axis of the ellipse) relative to the $x$-axis is given by

$$
\Phi=\frac{\phi_{R_{+}}+\phi_{R_{-}}}{2}
$$

Similarly, the water surface slope $(S)$ and the frictional shear stress $(T)$ can be divided into anticlockwise $\left(S_{+}, T_{+}\right)$and clockwise $\left(S_{-}, T_{-}\right)$rotating components in the same manner as the velocity. By introducing this notation, Eqn. (1) can be

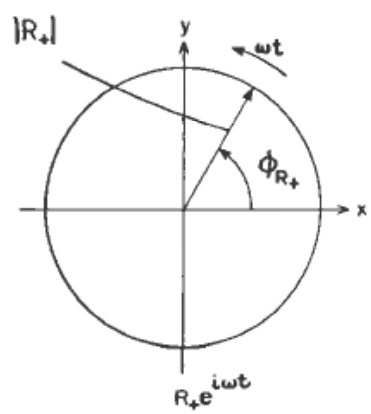

(a)

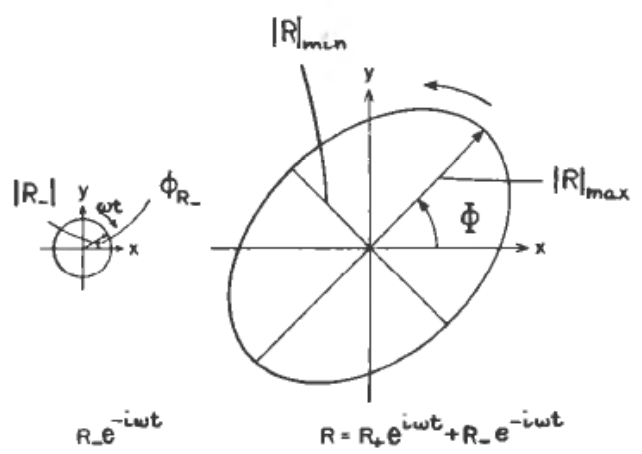

(c)

Figure 1. Current ellipse components: (a) anticlockwise component; (b) clockwise component; (c) resultant ellipse (after Prandle 1982). 
separated into independent anticlockwise and clockwise components. Outside the boundary layer the frictionless current is given by

$$
\begin{aligned}
& R_{\infty+}=\frac{\mathrm{i} g S_{+}}{\omega+f} \\
& R_{\infty-}=-\frac{\mathrm{i} g S_{-}}{\omega-f}
\end{aligned}
$$

The frictional shear stress will be modelled as

$$
\begin{aligned}
& T_{+}=\rho \varepsilon_{+} \frac{\partial R_{+}}{\partial z} \\
& T_{-}=\rho \varepsilon_{-} \frac{\partial R_{-}}{\partial z}
\end{aligned}
$$

where the kinematic eddy viscosities $\varepsilon_{+}$and $\varepsilon_{-}$associated with the anticlockwise and clockwise rotations, respectively, are assumed to be the same in both horizontal directions. $\varepsilon_{+}$and $\varepsilon_{-}$will be discussed below. By introducing this notation and the defect velocities

$$
\begin{aligned}
& R_{d+}=R_{+}-R_{\infty+} \\
& R_{d-}=R_{-}-R_{\infty-}
\end{aligned}
$$

Equation (1) can be separated into independent anticlockwise and clockwise components (since $R_{\infty+}$ and $R_{\infty}$ - are independent of $z$ ) given by

$$
\begin{aligned}
& \frac{\partial}{\partial z}\left(\varepsilon_{+} \frac{\partial R_{d+}}{\partial z}\right)-\mathrm{i}(\omega+f) R_{d+}=0 \\
& \frac{\partial}{\partial z}\left(\varepsilon_{-} \frac{\partial R_{d-}}{\partial z}\right)+\mathrm{i}(\omega-f) R_{d-}=0
\end{aligned}
$$

subject to the boundary conditions

$$
\begin{array}{lll}
R_{d+}=-R_{\infty+} & \text { at } & z=z_{0} \\
R_{d-}=-R_{\infty-} & \text { at } & z=z_{0}
\end{array}
$$

and

$$
R_{d+}, R_{d-} \rightarrow 0 \text { for } z \rightarrow \infty
$$

The flow is assumed to be in the rough turbulent regime, that is (Soulsby 1983)

$$
\frac{u_{* m} k}{v}>165
$$

In the roughness Reynolds number, $u_{v_{m}} k / v, v$ is the coefficient of kinematic viscosity of the fluid, $k$ is the Nikuradse's equivalent sand roughness, that is, the characteristic dimension of the physical roughness of the seabed, and $u_{s_{m}}$ is the maximum friction velocity on the seabed defined by

$$
u_{s_{m}}=\left(\frac{\tau_{0 \max }}{\rho}\right)^{1 / 2}=\left[\frac{\left(\tau_{x z}^{2}+\tau_{y z}^{2}\right)_{z=z_{0 \max }}^{1 / 2}}{\rho}\right]^{1 / 2}
$$


where $\tau_{0 \max }=\left|T_{z=z_{0 \max }}\right|$ is the magnitude of the maximum shear stress on the seabed. In the ocean $k$ may be very different than the local physical roughness would suggest. (23) is valid provided that the height of the roughness elements replaces $k$ if the seabed is not flat (see Soulsby (1983) for a closer discussion). Soulsby (1983) gives a detailed discussion about how $k$ is determined for various seabed conditions.

When the flow is assumed to be in the rough turbulent regime the seabed roughness elements are higher than in the (hypothetical) viscous sublayer. Since the eddy viscosity is of greater magnitude than the molecular viscosity close to the seabed, the shear stress due to the molecular viscosity has been neglected in the equations of motion.

For rough turbulent flow $z_{0}=k / 30$, and the boundary condition on the seabed is taken analogously to be that used for steady, unidirectional rough, fully turbulent flow based on laboratory experiments (Schlichting 1979).

In this study the following two-layer, time-invariant eddy viscosity model is proposed (which was originally used to model rough turbulent wave boundary layers in Myrhaug (1982))

$$
\begin{gathered}
\frac{\varepsilon_{i \pm}}{\kappa u_{*_{m}} \delta_{ \pm}}=\frac{1}{2}-\frac{1}{2}\left(\frac{z}{\delta_{ \pm}}-1\right)^{2} \text { for } z \leqslant \delta_{ \pm} \\
\frac{\varepsilon_{o \pm}}{\kappa u_{*_{m}} \delta_{ \pm}}=\frac{1}{2} \text { for } z>\delta_{ \pm}
\end{gathered}
$$

where $\kappa$ is von Karman's constant, $\delta_{+}$and $\delta_{-}$are the distances from the seabed where the overlap takes place. Note that $\delta_{+}$and $\delta_{-}$in this context are overlap heights and that the boundary layer thicknesses will be greater than $\delta_{+}$and $\delta_{-}$. The indexes $i$ and $o$ denote the inner and outer boundary layers, respectively. A Taylor expansion of (25) for small $z / \delta_{ \pm}$reduces to $\varepsilon_{i}=\kappa u_{*} z$, which automatically gives the correct logarithmic profile near the seabed. Different values are assigned to von Karman's constant depending on the problem which is under consideration. In laboratory experiments $\kappa=0.40$ or 0.41 is generally used, while atmospheric workers often opt for $\kappa=0.35$ (Soulsby 1983). In this study $\kappa=0.40$ has been selected, $\delta_{ \pm}$is taken to be

$$
\delta_{ \pm}=C \frac{u_{\star_{m}}}{\omega \pm f} ; \quad C=0.05
$$

since $u_{\iota_{m}} /(\omega \pm f)$ are characteristic lengths of the thicknesses of the boundary layer. The justification for using the factor $C=0.05$ in (27) is obtained from comparisons of model predictions and the experimental results from measurements of the current structure in a time-independent boundary layer under drifting pack ice (reported by McPhee and Smith (1976), see Myrhaug (1987)). This $C$-value will also be used here. The choice of $C$ determines the magnitude of the eddy viscosity in the outer layer, as well as the height of the overlap point. The proposed model for $\varepsilon_{+}$and $\varepsilon_{-}$seems to have a reasonably realistic behaviour. Both $\varepsilon_{+}$and $\varepsilon_{-}$reach a maximum at $z=\delta_{+}$ and $z=\delta_{-}$, respectively, and these values are kept constant throughout the boundary layer. Even more realistic behaviour would be obtained by letting $\varepsilon_{+}$and $\varepsilon_{-}$ decrease to zero as $z \rightarrow \infty$, as was done in the Businger and Arya (1974) and Long (1981) models. Businger and Arya (1974) used the following eddy viscosity model: $\varepsilon=\kappa u_{*_{m}} z \exp (-z / h)$, where $h$ is the boundary layer scale height. This model was 
suggested for a steady planetary boundary layer and was later expanded upon by Long (1981) to cover unsteady flow. Soulsby (1983) has discussed a solution based on the eddy viscosity distribution: $\varepsilon=\kappa u_{*_{m}} z$. However, the present eddy viscosity model seems to be a reasonable compromise between accuracy and simplicity and has the benefit over the linear profile of having one disposable constant which allows the model to be adjusted to the data. Simple eddy viscosity models have often proved capable of predicting the overall structure of measured current profiles reasonably well. More refined turbulence modelling using higher-order closure models is necessary to obtain a better prediction of the detailed turbulent structure. However, in order to produce better modelling of turbulence one should also have access to high-quality data from detailed measurements of the turbulent structure as well as the mean fluid velocities in idealized neutrally stable boundary layer flow.

The solutions of Eqns. (18) and (19) with the same eddy viscosity model as in (25) and (26), and subject to the boundary conditions in (20)-(22) were given in a different context in Myrhaug (1982) (see also Myrhaug, 1987), consequently the details will not be repeated here. The maximum friction velocity on the seabed is given by

$$
\begin{aligned}
u_{*_{m}}= & \frac{1}{2} \kappa\left\{\left(1-\xi_{0+}^{2}\right)\left[\chi_{+}\left(\xi_{0_{+}} ; u_{*_{m}}\right) \cdot \hat{\chi}_{+}\left(\xi_{0_{+}} ; u_{*_{m}}\right)\right]^{1 / 2}\right. \\
& \left.+\left(1-\xi_{0_{-}}^{2}\right)\left[\chi_{-}\left(\xi_{0_{-}} ; u_{*_{m}}\right) \cdot \hat{\chi}_{-}\left(\xi_{0_{-}} ; u_{*_{m}}\right)\right]^{1 / 2}\right\}
\end{aligned}
$$

where $\hat{\chi}_{ \pm}$denotes the complex conjugate of $\chi_{ \pm}$, which is given in the Appendix together with $\xi_{0 \pm}$. Equation (28) is an implicit equation for the determination of $u_{*_{m}}$. For a given seabed roughness length, free stream current velocity, frequency of oscillation and the Coriolis parameter, $u_{*_{m}}$ can be determined from Eqn. (28) by iteration.

\section{Comparison with measurements}

Our results will now be compared with the measurements made on the continental shelf south-west of Lands End, England reported by Pingree and Griffiths (1974). These measurements were taken under completely neutral conditions well away from the influence of local coastlines. The current metres were moored in water depths of $180 \mathrm{~m}$ more than $200 \mathrm{~km}$ from the mainland. For mooring no. 1 the measurements were made at $3 \cdot 5,7 \cdot 5,33.5$ and $98 \mathrm{~m}$ above the bottom. The semidiurnal frequency of oscillation and the Coriolis parameter were $\omega=0.000143 \mathrm{rad} / \mathrm{s}$ and $f=0.000108 \mathrm{rad} / \mathrm{s}$, respectively. The free stream amplitudes of anticlockwise and clockwise motion were $\left|R_{\infty}\right|=9.9 \mathrm{~cm} / \mathrm{s}$ and $\left|R_{\infty}-\right|=35.2 \mathrm{~cm} / \mathrm{s}$, respectively, $33.5 \mathrm{~m}$ above the bottom. The logarithmic boundary layer flow model was matched to the data for the lowest $33.5 \mathrm{~m}$ and the logarithmic flow model friction velocity $\left(u_{*}\right)$ and roughness $\left(z_{0}\right)$ parameters were determined. For the clockwise component the following parameters were found: $u_{*-}=1.2 \mathrm{~cm} / \mathrm{s}$ and $z_{0}=0.03 \mathrm{~cm}$ (with von Karman's constant $\kappa=0.4$ ). The friction velocity associated with the anticlockwise motion was $u_{*+}=0.3 \mathrm{~cm} / \mathrm{s}$. Thus the maximum friction velocity $u_{*_{m}}=u_{*_{+}}$ $+u_{*-}=1.5 \mathrm{~cm} / \mathrm{s}$. The assumed values of $\left|R_{\infty}-\right|,\left|R_{\infty}-\right|, \omega, f$ and $z_{0}$ give $u_{*_{m}}=$ $1.47 \mathrm{~cm} / \mathrm{s}$ according to Eqn. (28), which appears to be in very good agreement with the estimate from the measurements. In this case $u_{c_{m}} k / v$ is about 135 , i.e. the flow is in the transitional smooth to rough turbulent flow regime. Since this is fairly close the rough turbulent regime according to (23), our theoretical results are taken to be valid. 


\section{Conclusions}

This paper has presented an analytical theory describing the fluid motion in a turbulent oscillatory planetary boundary layer near a rough seabed. A two-layer time-invariant eddy viscosity is used to model the shear stress. The present eddy viscosity model is a reasonable compromise between accuracy and simplicity and offers one disposable constant which can determine the magnitude of the eddy viscosity in the outer layer, as well as the height of the overlap point. This permits the model to be adjusted to the data. The bottom shear stress is presented and a comparison is made with measurements of turbulent tidal planetary boundary layer flow on the continental shelf off the south-west of England, reported by Pingree and Griffiths (1974). The estimated bottom shear stress from these measurements agrees very well with the prediction made by our simple eddy viscosity model.

\section{ACKNOWLEDGEMENT}

The author would like to thank O. H. Slaattelid for modifying the computer programs.

\section{Appendix}

$\chi_{ \pm}$is a complex quantity with the dimension of velocity given by

$$
\chi_{ \pm}\left(\xi_{ \pm} ; u_{\oplus_{m}}\right)=R_{\infty \pm} \frac{\left(p_{ \pm}-\cos \lambda_{ \pm} \pi\right) P_{\lambda_{ \pm}}^{\prime}\left(\xi_{ \pm}\right)-P_{\lambda_{ \pm}}^{\prime}\left(-\xi_{ \pm}\right)}{\left(p_{ \pm}-\cos \lambda_{ \pm} \pi\right) P_{\lambda_{ \pm}}\left(\xi_{0 \pm}\right)+P_{\lambda_{ \pm}}\left(-\xi_{0 \pm}\right)}
$$

where

$$
P_{\lambda_{ \pm}}^{\prime}(x) \equiv \frac{d P_{\lambda_{ \pm}}(x)}{d x}=\frac{\lambda_{ \pm}+1}{x^{2}-1}\left[P_{\lambda_{ \pm}+1}(x)-x P_{\lambda_{ \pm}}(x)\right]
$$

$P_{\lambda_{ \pm}}(\xi)$ is the Legendre function of the first kind given in terms of the normalized variables

$$
\xi_{ \pm}=\frac{z}{\delta_{ \pm}}-1 \text { and } \xi_{0 \pm}=\frac{z_{0}}{\delta_{ \pm}}-1
$$

$\lambda_{ \pm}$is given by

$$
\lambda_{ \pm}=-\frac{1}{2}(1-\alpha) \mp \mathrm{i} \frac{\beta}{\alpha}
$$

where

$$
\begin{aligned}
& \alpha=\left(\frac{1}{2}\left(1+\left(1+16 \beta^{2}\right)^{1 / 2}\right)\right)^{1 / 2} \\
& \beta=\frac{2 \delta_{ \pm}(\omega \pm f)}{\kappa u_{r_{m}}}=2 \frac{C}{\kappa}
\end{aligned}
$$

$p_{ \pm}$is given by

$$
p_{ \pm}=\frac{\eta_{ \pm}(1 \pm \mathrm{i}) \delta_{ \pm}\left(\cos \lambda_{ \pm} \pi-1\right)+\left(\cos \lambda_{ \pm} \pi+1\right) q_{ \pm}}{\eta_{ \pm}(1 \pm \mathrm{i}) \delta_{ \pm}+q_{ \pm}}
$$


where

$$
\begin{aligned}
\eta_{ \pm} & =\left(\frac{\omega \pm f}{\kappa u_{*_{m}} \delta_{ \pm}}\right)^{1 / 2} \\
\eta_{+} \delta_{+} & =\eta_{-} \delta_{-}=\sqrt{ }(C / \kappa) \\
q_{ \pm} & =\frac{1}{4 \pi^{2}} \lambda_{ \pm}^{2} \sin \lambda_{ \pm} \pi\left[\Gamma\left(\frac{\lambda_{ \pm}}{2}\right) \Gamma\left(\frac{1}{2}-\frac{\lambda_{ \pm}}{2}\right)\right]^{2}
\end{aligned}
$$

where $\Gamma$ is the Gamma-function.

It is seen that $\lambda$ and $p$ are both constants which only depend on $C / \kappa$.

\section{REFERENCES}

Businger, J. A. and ARYA, S. P. S. (1974). Height of the mixed layer in the stably stratified planetary boundary layer. Advances in Geophysics (Academic Press, New York), pp. 73-92.

Davies, A. M. (1985). On determining current profiles in oscillatory flows. Applied Mathematical Modelling, 9, 419-428.

JENKINS, A. D. (1987). Wind and wave induced currents in a rotating sea with depth-varying eddy viscosity. Journal of Physical Oceanography. 17, 938-951.

King, H. L., Davies, A. G., and SoulsBy, R. L. (1985). A numerical model of the turbulent boundary layer beneath surface waves and tides. Institute of Oceanographic Sciences, Report No. 196, Wormley, Godalming, Surrey, England, 90 pp.

LONG, C. E. (1981). A simple model for time-dependent stably stratified turbulent boundary layers. Dept. of Oceanography, Spec. Rep. 95, University of Seattle, Seattle, Washington, $170 \mathrm{pp}$.

McPheE, M. G. and Smith, J. D. (1976). Measurements of the turbulent boundary layer under pack ice. Journal of Physical Oceanography. 6, 696-711.

MyrhaUG, D. (1982). On a theoretical model of rough turbulent wave boundary layers. Ocean Engineering, 9, 547-565.

MYrHAUG, D. (1987). Comparison of a simple planetary boundary layer with measurements of a turbulent boundary layer under pack ice. Continental Shelf Research, 7, 135-148.

PingReE, R. D. and GrifFiths, D. K. (1974). The turbulent boundary layer on the continental shelf. Nature, 250, 720-722.

PrandLe, D. (1982). The vertical structure of tidal currents. Geophys. Astrophys. Fluid Dynamics, 22, 29-49.

SCHLICHTING, H. (1979). Boundary-Layer Theory. 7th Edition (McGraw-Hill, New York)

SouLSBY, R. L. (1983). The bottom boundary layer of shelf seas, in (Physical Oceanography of Coastal and Shelf Seas (edited by B. Johns) (Elsevier, New York), pp. 189-266.

Sverdrup, H. U. (1927). Dynamic of tides on the North Siberian Shelf, Geofysiske Publikasjoner $4,75 \mathrm{pp}$. 